\title{
Revisited clinical implication of 99mTc-Sestamibi scan for staging Multiple Myeloma as an affordable alternative modality: A Case report
}

\author{
Afroza Naznin', Azmal K Sarker ${ }^{1,2}$, Hosne Ara Rahman', Samira Sharmin', Jamiul Hossain' \\ ${ }^{1}$ Institute of Nuclear Medicine and Allied Sciences, Mitford, Sir Salimullah Medical College Hospital campus, Dhaka. \\ ${ }_{2}^{2}$ Institute of Nuclear Medical Physics, Atomic Energy Research Establishment, Savar, Dhaka.
}

Correspondence Address: Afroza Naznin, Institute of Nuclear Medicine and Allied Sciences, Mitford, Dhaka, afroza.naznin@yahoo.com

\begin{abstract}
Background: Staging of multiple myeloma (MM) requires quantification of lesions and serum biomarkers. Sestamibi (MIBI) scan can detect medullary and extra-medullary lesions whereas pathological fracture of MM when associated with osteoblastic activity which can be imaged by methyl diphosphonate (MDP).
\end{abstract}

Patients and methods: This case report is of a biopsy proven case of $\mathrm{MM}$ with serum biomarkers inconclusive for staging. With evidence of multiple pathological rib fractures on conventional radiography, the patient was sent for MDP scan as she was unable to afford any other modality for whole body survey. A complimentary MIBI scan was therefore done.

Results: MDP scan revealed multiple osteoblastic lesions in spine and ribs. The MIBI scan was conclusive for stage II active disease.

Conclusions: MIBI scan added to diagnostic information and aided an inexpensive staging. MIBI scan can be an affordable work-up tool for MM. Key wards: Multiple Myeloma, ${ }^{99 \mathrm{mTc}-M I B I}$ Scan.

Bangladesh J. Nucl. Med. Vol. 22 No. 2 July 2019

Doi: https://doi.org/10.3329/bjnm.v22i2.51769

\section{INTRODUCTION}

Multiple myeloma (MM) is a neoplastic disorder of plasma B-cells which is characterized by bone marrow infiltration resulting in over production of monoclonal immunoglobulins. It represents about $10 \%$ of all hematological malignancies and $1 \%$ of all cancers. Standard investigations for diagnosis of MM includes a complete blood count, serum biochemistry, serum and urine electrophoresis, and bone marrow biopsy which is considered as gold standard (1). Osteolytic bone lesions are seen in up to $90 \%$ of patients and X-ray radiography have been conventionally used for assessing the extent of bony involvement, but with limited sensitivity (2). On the other hand, specificity and sensitivity of whole body planar bone scintigraphy with Technetium-99m methylene diphosphonate (99mTc MDP) for initial diagnosis, follow-up and evaluation of bone pain is even lower compared to conventional radiography primarily due to the osteoblastic dysfunction present in myeloma (3). ${ }^{99 \mathrm{mTc}}$ Sestamibi (99mTc MIBI) imaging is an alternative nuclear imaging modality that accurately represents myeloma activity in the bone marrow with high sensitivity and specificity (4). The ability of MIBI to concentrate in multiple myeloma cells has been under investigation since 1996 and the results led to growing clinical interest in its role in the work-up of MM patients (5).

\section{CASE REPORT}

A 63-year-old female with referral from oncologist came to Institute of Nuclear Medicine \& Allied Sciences (INMAS), Mitford, Dhaka in March'2019 for bone scintigraphy. Her chief complaint was bone pain for several months. On routine investigation, she was found to have hypercreatinemia $(2.4 \mathrm{mg} / \mathrm{dl}$, whereas normal level $0.6-1.3 \mathrm{mg} / \mathrm{dl})$ and mild hypercalcemia $(11 \mathrm{mg} / \mathrm{dl}$, whereas normal level 8.4-10.2 mg/dl). Peripheral blood film revealed combined deficiency anemia as the red blood corpuscles were macrocytic as well as microcytic \&hypochromic. Urine examination for Bence Jones protein was negative. Her bone marrow biopsy showed features of plasma cell dyscrasia compatible with multiple myeloma. Protein electrophoresis was however indeterminate, showing pattern of beta fraction doubtful. Chest X-ray showed multiple rib fractures on right side. At this point of work-up the patient was unable to afford whole body skeletal survey and was therefore referred for bone scan. With this scenario in hand, we performed $99 \mathrm{mTc}$ MDP bone scan of the patient and found multiple focal areas of increased radiotracer concentration in thoracic \& lumbar regions of spine with mild spinal deformities, as well as in multiple ribs of both sides with some of the foci in linear fashion suggesting pathological fracture (Figure 1). 
Considering the diagnostic constraint in this case, we decided to explore the tumor seeking capacity of MIBI and scanned the patient with Tc-99m MIBI after three days. Whole body planar 99mTc MIBI scan revealed mixed pattern of marrow uptake consisting of multiple (at least five) focal areas along with diffuse areas of moderately intense (more than skeletal muscle but less than myocardium) uptake in the thoracolumbar vertebrae, humeri and femora. In addition, extra- medullary intense uptake was seen in liver; altogether suggestive of stage II active disease (Figure 2). To check and compare with a physiological reference distribution of tracer, a planer scan was performed on a consenting volunteer injected with $99 \mathrm{mTc}$ MIBI for other valid clinical indication.

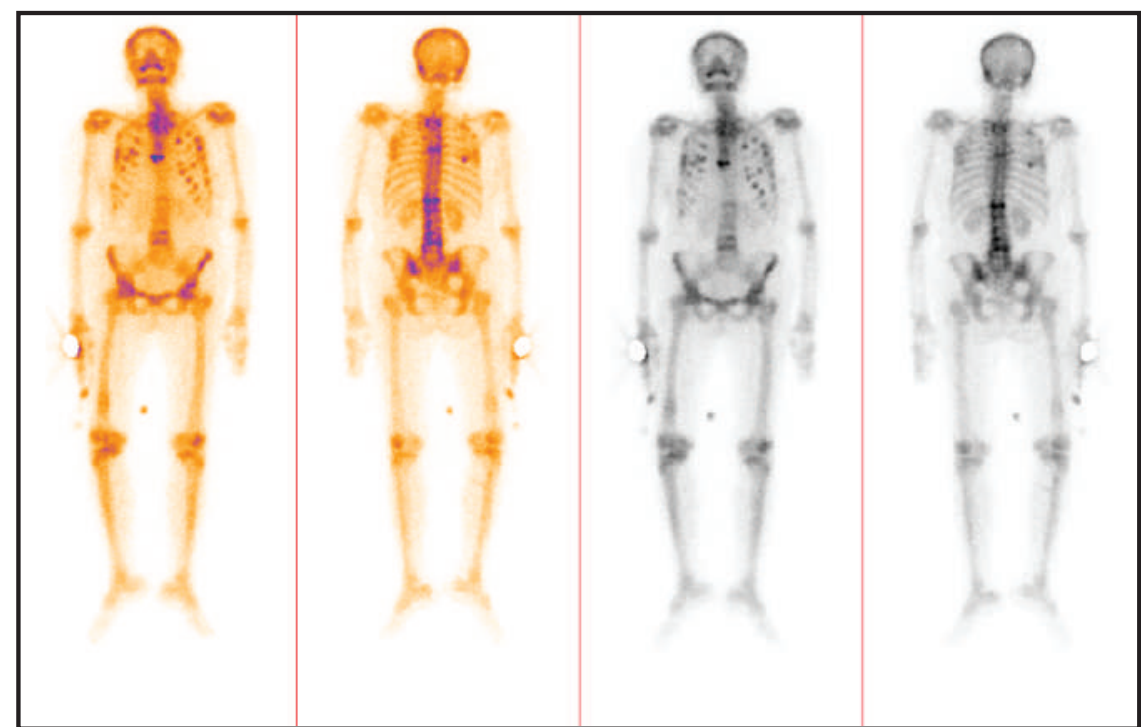

Figure 1: 99mTc-MDP whole body planar scan in a 63 years old female. There are osteoblastic lesions in thoraco-lumbar spine and in multiple ribs on both sides. There is spinal deformity. The linear arrangement and perpendicularity of rib lesions suggest likelihood for fracture.

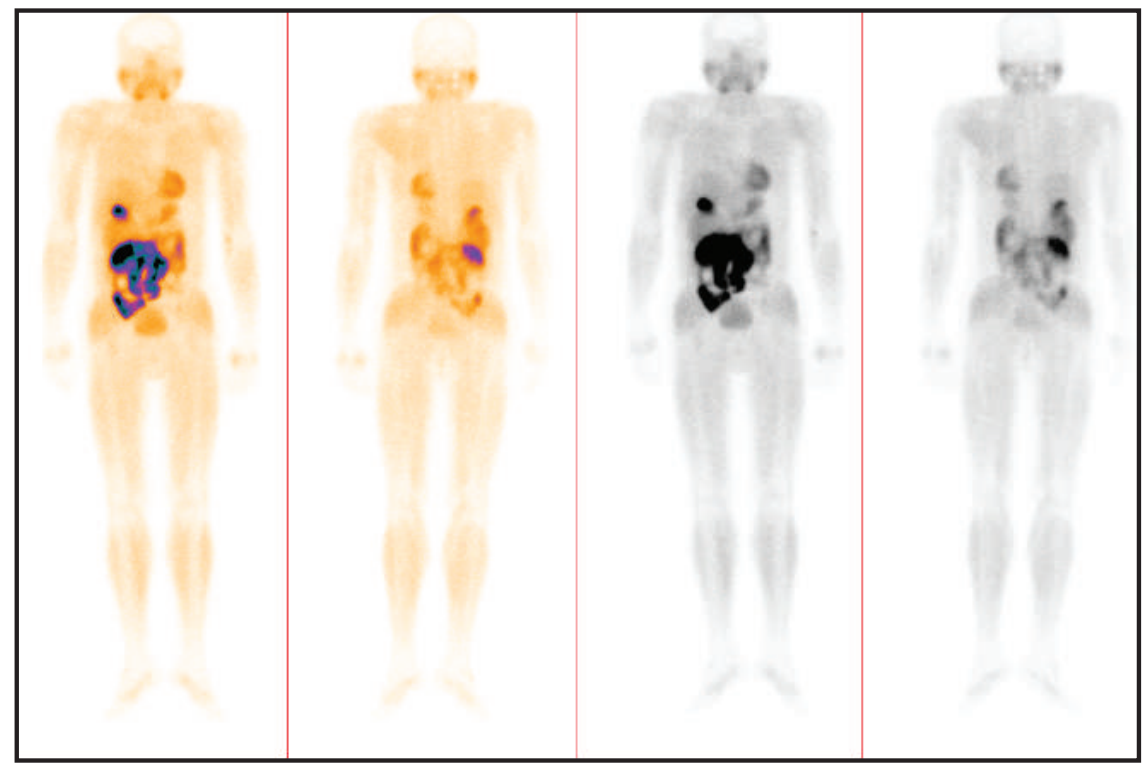

Figure 2: 99mTc-MIBI whole body planar scan in the same 63 years old female. There is diffuse intense uptake throughout the spine, pelvic bones, scapulae, both humeri and both femora with focal areas of uptake in sternum, multiple vertebrae and right femur. The uptake pattern is combined diffuse and focal pattern (D+F) as described by Pace et al (6). Extra-skeletal diffuse activity is seen in salivary gland, neck lymph nodes, thyroid, liver, spleen, intestine and kidneys. The extent of marrow uptake reaches up to distal epiphysis of left humerus and right femur (E score 3 ) whereas the intensity of uptake is more than muscle but less than the myocardium (I score 3) yielding a summed score of 6 that indicates an active disease with poor prognosis, as described by Giovanella et al. (7). 


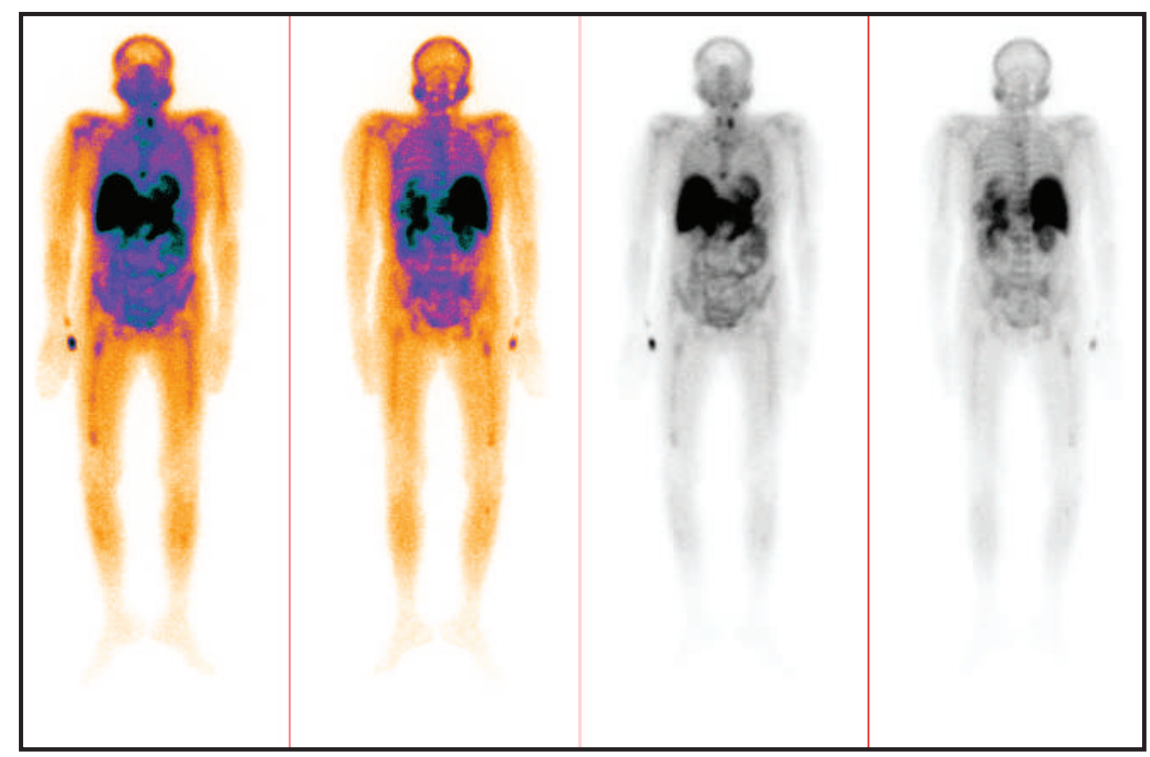

Figure 3: 99mTc-MIBI whole body planar scan in a consenting volunteer who was injected with radiotracer for clinically indicated SPECT myocardial perfusion imaging, imaged for visual comparison of physiological tracer distribution. Intense uptake is seen in gall bladder and intestines; moderate uptake in salivary glands, myocardium, liver and skeletal muscles; faint uptake in spleen, kidneys, urinary tract and thyroid. There is no osseous uptake at all.

\section{DISCUSSION}

\section{Clinical manifestations of multiple myeloma}

MM results from clonal proliferation of malignant plasma cells in the bone marrow resulting in overabundance of aberrant antibodies. Complications of MM include hypercalcemia, renal failure, anemia and osteolytic lesions, all known as the CRAB criteria. Advanced stage MM may present with spinal cord compression due to tumor mass and pathological fracture of bone (8) owing to bone destruction in MM which in turn is attributed to increased osteoclastic bone reabsorption along with a reduced osteoblastic activity. Up to $90 \%$ of patients may develop osteolytic lesions during the course of the disease irrespective of initial skeletal involvement. Lesions typically occur in the axial skeleton and proximal areas of arms and legs, but can involve all bones(9). Extra-medullary lesions may occur in $7-18 \%$ cases of MM (10).

\section{Transmission imaging modalities for multiple myeloma work-up}

The updated Durie-Salmon PLUS system for MM staging, which was found to be determinative of treatment response and survival outcome incorporates lesion quantification from whole body imaging survey in addition to serum biomarkers (11). Historically the Mirels score on conventional radiography (CR) predicted risk of fracture and guided to select between radiotherapy or preventive osteosynthesis as an intervention in patients with MM (12). However, CR has failed to differentiate between active and residual nature of osteolysis as well as it failed to identify MM infiltration to bone marrow and soft tissue (13). CR has a long known drawback of false-negative rate of up to $70 \%$ owing to its ability of detecting lytic lesion only when $30-50 \%$ of the bone mineral density is already lost (1). On contrary, an early, accurate and quantifiable estimate of decalcification is obtainable from computed tomography (CT), magnetic resonance imaging (MRI) and Dual energy $x$ ray absorptiometry (DEXA) (12). The higher sensitivity of whole body CT is however outweighed by its cost and radiation burden to preclude its acceptance as a screening modality (1).Though MRI can provide assessment of tumor burden by identifying $\mathrm{MM}$ associated fatty infiltration of bone marrow and the guidelines originated from advanced world favor use of whole body MRI (4), the cost and availability of this modality hinders its suitability in Bangladesh (14).

\section{Clinical applicability of emission imaging modalities in multiple myeloma}

Bone scintigraphy using $99 \mathrm{mTc}$ MDP is accused to miss up to $50 \%$ bone lesions and then has been disfavored in recent influential recommendation (15). Nonetheless, it 
can pick the osteoblastic activity of MM in response to a pathological compression fracture of vertebral body or pelvic insufficiency fracture (1) while a recent series from limited resource set-up observes $84 \%$ sensitivity of MDP scan for lesion detection (15).

Hybrid Positron Emission Tomography-Computed Tomography (PET-CT) with F-18-Fluoro-deoxy glucose (FDG) is increasingly proven useful for initial diagnosis, for detection of medullary and extra-medullary disease, for post therapy assessment of response and for differentiation of relapsed active disease from residual morphological scars (16). Accessibility and affordability of F-18-FDG PET-CT is however yet to achieve in Bangladesh (17).

99mTc MIBI scan in comparison to F-18-FDG PET is affordable, technically less demanding and has wider availability in Bangladesh through the INMASes. MIBI positivity is a surrogate of bone marrow plasma cell infiltration and cytogenetic abnormality, and thus can guide bone marrow biopsy $(6,7)$ especially in cases of focal infiltration where biopsy has a high chance to miss

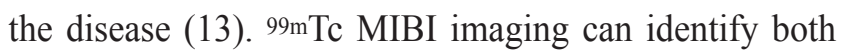
skeletal and extra-medullary soft tissue lesions of MM with the overall sensitivity of about $92 \%$ and specificity $96 \%$. It has been found to be superior to PET/CT for the visualization of diffuse disease and can even show indolent or very low level myeloma which is not detectable on PET/CT $(1,18)$. Compared to MRI, Tc-99m MIBI underestimates the extent of bone marrow infiltration in the spine, skull and long bones but shows superiority in detecting lytic lesions in the ribcage. Besides, it is also able to predict overall disease prognosis better than MRI. Moreover, MIBI scan unlike MRI is less time consuming, more comfortable for the patient, can achieve image of all important bone compartments in one single examination $(4,19)$ and therefore cheaper than whole body MRI.

Patterns for whole body distribution of 99mTc-MIBI in MM was described, classified and correlated with advancing stage by Pace et al (6). Baseline patterns of MIBI uptake were found to be correlated with overall survival $(13,19)$. Moreover, the subjective categorization of diffuse lesion in Durie-Salmon PLUS staging system was overcome by the extent and intensity score of Pace et al (6) which has been further elaborated by Giovanelle et al (7). $99 \mathrm{mTc}$-MIBI planar imaging however is likely to miss the para-cardiac, paravertebral and cervical lesions which is better appreciated by SPECT. False positive detection of physiological skeletal muscle activity can be discerned by hybrid SPECT-CT (18). Finally, a pooled meta-analysis of 17 studies comprising of 575 patients revealed comparable diagnostic performance of 18-F-FDG-PET-CT, MIBI Scan and MRI for detection of bone disease (20).

\section{CONCLUSIONS}

Traditionally, multiple myeloma has been considered as a disease where radionuclide scintigraphy plays a very limited role. Whole body CT, MRI or PET-CT scan for skeletal survey is not always available or cost-effective in context of Bangladesh. Utilization of a radiotracer like MIBI which can be availed readily even in our limited resource country might bring positive effect to multiple myeloma patient management.

\section{REFERENCES}

1. Healy CF, Murray JG, Eustace SJ, Madewell J, O'gorman PJ, O'sullivan P. Multiple myeloma: a review of imaging features and radiological techniques. Bone marrow research. 2011;2011.

2. Smith A, Wisloff F, Samson D, UK Myeloma Forum, Nordic Myeloma Study Group and British Committee for Standards in Haematology. Guidelines on the diagnosis and management of multiple myeloma 2005. British journal of haematology. 2006 Feb;132(4):410-51.

3. Ludwig H, Kumpan W, Sinzinger H. Radiography and bone scintigraphy in multiple myeloma; a comparative analysis. The British journal of radiology. 1982 Mar;55(651):173-81.

4. Khalafallah AA, Snarski A, Heng R, Hughes R, Renu S, Arm J, Dutchke R, Robertson IK, To LB. Assessment of whole body MRI and sestamibi technetium- $99 \mathrm{~m}$ bone marrow scan in prediction of multiple myeloma disease progression and outcome: a prospective comparative study. BMJ open. 2013 Jan 1;3(1).

5. Jonsson C, Jacobsson H. Accumulation of 99m Tc-MIBI in bone marrow. Annals of nuclear medicine. 1996 Sep 1;10(3):281-5.

6. Palmer IM. Biodiversity and ecosystem processes. Ambio. 1997 Dec;26(8).

7. Giovanella L, Taborelli M, Ceriani L, Zucca E, Cavalli F, Delaloye AB. $99 \mathrm{mTc}$-sestamibi imaging and bone marrow karyotyping in the assessment of multiple myeloma and MGUS. Nuclear medicine communications. 2008 Jun 1;29(6):535-41.

8. Choudhury S, Sultana TA, Islam MS, Islam MA, Khanam PA. Multiple Myeloma-A hospital based cross sectional study in Bangladesh. Journal of the Asiatic Society of Bangladesh, Science. 2012;38(2):189-98.

9. Yaccoby S. Advances in the understanding of myeloma bone disease and tumour growth. British journal of haematology. 2010 May;149(3):311-21.

10. Bladé J, Fernandez de Larrea C, Rosinol L, Cibeira MT, Jiménez R, 
Powles R. Soft-tissue plasmacytomas in multiple myeloma: incidence, mechanisms of extramedullary spread, and treatment approach. Journal of Clinical Oncology. 2011 Oct 1;29(28):3805-12.

11. Filonzi G, Mancuso K, Zamagni E, Nanni C, Spinnato P, Cavo M, Fanti S, Salizzoni E, Bazzocchi A. A comparison of different staging systems for multiple myeloma: can the MRI pattern play a prognostic role?. American Journal of Roentgenology. 2017 Jul;209(1):152-8.

12. Vanicek J, Szturz P, Rehak Z, Kianicka B, Bulik M. Benefits of Individual Imaging Methods for Diagnosis and Monitoring of Activity of Multiple Myeloma. Klinicka onkologie: casopis Ceske a Slovenske onkologicke spolecnosti. 2012;25(3):166-72.

13. Bacovsky J, Myslivecek M, Scudla V, Koranda P, Buriankova E, Minarik J, Pika T, Zapletalova J. Tc-99m MIBI scintigraphy in multiple myeloma: Prognostic value of different Tc-99m MIBI uptake patterns. Clinical Nuclear Medicine. 2010 Sep 1;35(9):667-70.

14. Chowdhury AA, Hassan MS, Chowdhury AR, Farahnaj S, Haque MI, Chowdhury M. Price of Imaging Tests and Access of Patients to Modern Health Care Facilities across the Developing and Better-Off Countries: A Comparative Study. Resonance. 2016;88(106.34):106-34.
15. Sadeghi MV, Sedaghat $\mathrm{S}$. Is $99 \mathrm{~m}$ Tc-methylene diphosphonate bone scintigraphy a sensitive method for detecting bone lesions in multiple myeloma?. Caspian Journal of Internal Medicine. 2018;9(2):140.

16. Jamet B, Bailly C, Carlier T, Touzeau C, Nanni C, Zamagni E, Barré L, Michaud AV, Chérel M, Moreau P, Bodet-Milin C. Interest of pet imaging in multiple myeloma. Frontiers in medicine. 2019 Apr 9;6:69.

17. Lee DS, Lee YS, Lee JS, Suh MS. Promotion of Nuclear Medicine-Related Sciences in Developing Countries. Nuclear medicine and molecular imaging. 2019 Apr 1;53(2):73-82.

18. Luthra K, Bhave A, Lele RD. Tc 99m Sestamibi Scanning in Multiple Myeloma--a New look with SPECT-CT. J Assoc Physicians India. 2014 Sep;62(9):801-12.

19. Fonti R, Pace L, Cerchione C, Catalano L, Salvatore B, De Luca S, Pane F, Salvatore M, Del Vecchio S. 18F-FDG PET/CT, 99mTc-MIBI, and MRI in the prediction of outcome of patients with multiple myeloma: a comparative study. Clinical Nuclear Medicine. 2015 Apr 1;40(4):303-8

20. Weng WW, Dong MJ, Zhang J, Yang J, Xu Q, Zhu YJ, Liu NH. A systematic review of MRI, scintigraphy, FDG-PET and PET/CT for diagnosis of multiple myeloma related bone disease-which is best?. Asian Pacific Journal of Cancer Prevention. 2014;15(22):9879-84. 